H. Umemura and H. Watanabe

Nagoya Math. J.

Vol. 151 (1998), 1-24

\title{
SOLUTIONS OF THE THIRD PAINLEVÉ EQUATION I
}

\author{
HIROSHI UMEMURA AND HUMIHIKO WATANABE
}

\begin{abstract}
We classify transcendental classical solutions of the third Painlevé equation. This result combined with the list of algebraic solutions in [11] gives a complete table of classical solutions of the third Painlevé equation.
\end{abstract}

\section{$\S 0$. Introduction}

In this paper we determine transcendental classical solutions of the third Painlevé equation by applying the method of our previous papers [23] and [25]. According to Okamoto [15], the third Painlevé equation is written as the following system $\tilde{S}(\mathbf{v})$ of ordinary differential equations of first order for variable $T$ and unknowns $Q$ and $P$ :

$$
\tilde{S}(\mathbf{v}) v \quad\left\{\begin{array}{l}
T \frac{d Q}{d T}=4 Q^{2} P-2 T Q^{2}+\left(2 v_{1}-1\right) Q+2 T \\
T \frac{d P}{d T}=-4 Q P^{2}+4 T Q P-\left(2 v_{1}-1\right) P+\left(v_{1}+v_{2}\right) T
\end{array}\right.
$$

where $\mathbf{v}=\left(v_{1}, v_{2}\right)$ denotes a vector in $\mathbf{C}^{2}$. Here we notice that in our notation the vector $\mathbf{v}=\left(v_{1}, v_{2}\right) \in \mathbf{C}^{2}$ corresponds to the vector $\left(-\theta_{0},-\theta_{\infty}\right)$ in Okamoto's notation (cf. [15]). As is explained in [15], if we set

$$
t=T^{2}, \quad p=T^{-1} P, \quad q=T Q,
$$

then we obtain a new system $S(\mathbf{v})$ of differential equations

$$
S(\mathbf{v}) \quad\left\{\begin{array}{l}
t \frac{d q}{d t}=2 q^{2} p-q^{2}+v_{1} q+t \\
t \frac{d p}{d t}=-2 q p^{2}+2 q p-v_{1} p+\frac{1}{2}\left(v_{1}+v_{2}\right) .
\end{array}\right.
$$

Therefore the determination of transcendental classical solutions for $S(\mathbf{v})$ is equivalent to that for $\tilde{S}(\mathbf{v})$. In this paper we treat the system $S(\mathbf{v})$ mainly, and, if necessary, refer to the original system $\tilde{S}(\mathbf{v})$.

Received April 16, 1996. 


\section{$\S 1$. Preliminaries and principal results}

Following Okamoto [15], we summarize some basic properties of the following system $S(\mathbf{v})$ of ordinary differential equations of first order for variable $t$ and unknowns $q$ and $p$ :

$$
S(\mathbf{v}) \quad\left\{\begin{array}{l}
t \frac{d q}{d t}=2 q^{2} p-q^{2}+v_{1} q+t \\
t \frac{d p}{d t}=-2 q p^{2}+2 q p-v_{1} p+\frac{1}{2}\left(v_{1}+v_{2}\right)
\end{array}\right.
$$

where $\mathbf{v}=\left(v_{1}, v_{2}\right)$ denotes a vector in $\mathbf{C}^{2}$. We define four affine transformations $s_{i}(i=1,2,3,4)$ of the complex vector space $\mathbf{C}^{2}$ by $s_{1}(\mathbf{v})=\left(v_{2}, v_{1}\right)$, $s_{2}(\mathbf{v})=\left(-v_{2},-v_{1}\right), s_{3}(\mathbf{v})=\left(v_{2}+1, v_{1}-1\right), s_{4}(\mathbf{v})=\left(-v_{2}+1,-v_{1}+1\right)$. We have $s_{i}^{2}=1(i=1,2,3,4), s_{i} s_{j}=s_{j} s_{i}(i=1,3, j=2,4)$, where 1 denotes the identity transformation of $\mathbf{C}^{2}$. Let $\mathbf{G}$ be the subgroup generated by the four $s_{i}$ 's in the group of all affine transformations of $\mathbf{C}^{2}$. Let $\Gamma$ be the subset of $\mathbf{C}^{2}$ that consists of all the vectors $\mathbf{v}=\left(v_{1}, v_{2}\right)$ subject to the following conditions:

(i) $\Re\left(v_{1}-v_{2}\right) \geq 0$;

(ii) $\Re\left(v_{1}+v_{2}\right) \geq 0$;

(iii) $\Re\left(v_{1}-v_{2}-1\right) \leq 0$;

(iv) $\Re\left(v_{1}+v_{2}-1\right) \leq 0$;

(v) $\Im\left(v_{1}-v_{2}\right) \geq 0$ if $\Re\left(v_{1}-v_{2}\right)=0$;

(vi) $\Im\left(v_{1}+v_{2}\right) \geq 0$ if $\Re\left(v_{1}+v_{2}\right)=0$;

(vii) $\Im\left(v_{1}-v_{2}\right) \leq 0$ if $\Re\left(v_{1}-v_{2}-1\right)=0$;

(viii) $\Im\left(v_{1}+v_{2}\right) \leq 0$ if $\Re\left(v_{1}+v_{2}-1\right)=0$.

Here $\Re(v)$ and $\Im(v)$ denote the real and imaginary parts respectively of a complex number $v$. Then we have the following:

Lemma 1.1. The set $\Gamma$ is a fundamental region of $\mathbf{C}^{2}$ for the group $\mathbf{G}$.

We can prove the lemma by the same argument as in the proof of Lemma 3.1 in [23]. So we omit the proof. 
Now we introduce four subsets $W_{1}, W_{2}, D_{1}, D_{2}$ of $\mathbf{C}^{2}$ as follows:

$$
\begin{aligned}
& W_{1}=\left\{\mathbf{v} \in \mathbf{C}^{2} \mid v_{1}+v_{2} \in 2 \mathbf{Z}\right\} \cup\left\{\mathbf{v} \in \mathbf{C}^{2} \mid v_{1}-v_{2} \in 2 \mathbf{Z}\right\} \\
& W_{2}=\left\{\mathbf{v} \in \mathbf{C}^{2} \mid v_{1}+v_{2}-1 \in 2 \mathbf{Z}\right\} \cup\left\{\mathbf{v} \in \mathbf{C}^{2} \mid v_{1}-v_{2}-1 \in 2 \mathbf{Z}\right\} \\
& D_{1}=\left\{\mathbf{v} \in \mathbf{Z}^{2} \mid v_{1}+v_{2} \in 2 \mathbf{Z}\right\} \\
& D_{2}=\left\{\mathbf{v} \in \mathbf{Z}^{2} \mid v_{1}+v_{2}-1 \in 2 \mathbf{Z}\right\} .
\end{aligned}
$$

Here we regard the additive group $\mathbf{Z}^{2}$ as a subgroup of the additive group $\mathbf{C}^{2}$. The four subsets above are $\mathbf{G}$-invariant. The subset $\Gamma \cap W_{1}=\{\mathbf{v} \in \Gamma \mid$ $v_{1}-v_{2}=0$ or $\left.v_{1}+v_{2}=0\right\}$ is a fundamental region of $W_{1}$ for $\mathbf{G}$. The subset $\Gamma \cap W_{2}=\left\{\mathbf{v} \in \Gamma \mid v_{1}-v_{2}-1=0\right.$ or $\left.v_{1}+v_{2}-1=0\right\}$ is a fundamental region of $W_{2}$ for $\mathbf{G}$. The set $D_{1}$ is the orbit of the origin $\mathbf{0}$ of $\mathbf{C}^{2}$ by $\mathbf{G}$ (i.e., $D_{1}=\mathbf{G} \cdot \mathbf{0}$ ), and the set $D_{2}$ is the orbit of a point $(1,0) \in \mathbf{C}^{2}$ by $\mathbf{G}$ (i.e., $\left.D_{2}=\mathbf{G} \cdot(1,0)\right)$.

Let $\Sigma(\mathbf{v})$ be the set of solutions $(p, q)$ of $S(\mathbf{v})$. We set $\Sigma=\cup_{\mathbf{v}} \Sigma(\mathbf{v})$ (the disjoint union). We introduce four birational transformations $\sigma_{i}(i=$ $1,2,3,4)$ of the set $\Sigma$ as follows (see [15]): For $(p, q) \in \Sigma(\mathbf{v})$,

(i) we define $\sigma_{1}(p, q) \in \Sigma\left(s_{1}(\mathbf{v})\right)$ by

$$
\sigma_{1}(p, q)=\left(p, q+\frac{v_{1}-v_{2}}{2(p-1)}\right) \quad \text { if } v_{1}-v_{2} \neq 0
$$

and

$$
\sigma_{1}(p, q)=(p, q) \quad \text { if } v_{1}-v_{2}=0
$$

(ii) we define $\sigma_{2}(p, q) \in \Sigma\left(s_{2}(\mathbf{v})\right)$ by

$$
\sigma_{2}(p, q)=\left(p, q+\frac{v_{1}+v_{2}}{2 p}\right) \quad \text { if } v_{1}+v_{2} \neq 0,
$$

and

$$
\sigma_{2}(p, q)=(p, q) \quad \text { if } v_{1}+v_{2}=0
$$

(iii) we define $\sigma_{3}(p, q) \in \Sigma\left(s_{3}(\mathbf{v})\right)$ by

$$
\sigma_{3}(p, q)=\left(-\frac{1}{t} q^{2} p-\frac{v_{1}+v_{2}}{2 t} q, \frac{t}{q}\right)
$$


(iv) we define $\sigma_{4}(p, q) \in \Sigma\left(s_{4}(\mathbf{v})\right)$ by

$$
\sigma_{4}(p, q)=\left(1+\frac{1}{t} q^{2}(p-1)+\frac{v_{1}-v_{2}}{2 t} q,-\frac{t}{q}\right) .
$$

The definitions above are well-defined by the following facts: for each $(p, q) \in \Sigma(\mathbf{v})$

(i) $p-1 \neq 0$ if $v_{1}-v_{2} \neq 0$;

(ii) $p \neq 0$ if $v_{1}+v_{2} \neq 0$;

(iii) $q \neq 0$.

We can easily verify the three assertions above if we compare the similar parts in previous papers [23] and [25]. Therefore we omit the proofs.

Let $\mathbf{G}_{*}$ be the subgroup generated by the four $\sigma_{i}$ 's $(i=1,2,3,4)$ in the group of all bijections of the set $\Sigma$. The group $\mathbf{G}_{*}$ consists of birational transformations of $\Sigma$. There exists a surjective group morphism $f$ of $\mathbf{G}_{*}$ onto $\mathbf{G}$ such that $f\left(\sigma_{i}\right)=s_{i}(i=1,2,3,4)$. Let $\pi$ be the natural projection of $\Sigma$ onto $\mathbf{C}^{2}$ defined by $\pi(p, q)=\mathbf{v}$ for $(p, q) \in \Sigma(\mathbf{v})\left(\mathbf{v} \in \mathbf{C}^{2}\right)$. Then the following diagram is commutative for every $\gamma \in \mathbf{G}_{*}$ :

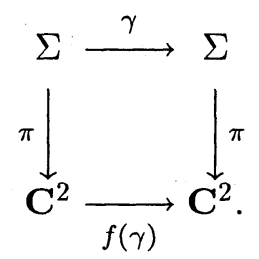

Remark 1.1. Our notation above is slightly different from Okamoto's in [15]. For example, our vector $\mathbf{v}=\left(v_{1}, v_{2}\right)$ in $\mathbf{C}^{2}$ corresponds to the vector $\left(-\theta_{0},-\theta_{\infty}\right)$ in his notation. Moreover, the groups $\mathbf{G}$ and $\mathbf{G}_{*}$ defined above are proper subgroups of the groups $\mathbf{G}$ and $\mathbf{G}_{*}$ in [15], respectively.

In [23], we defined a classical solution, an algebraic solution, etc. of the system $S(\mathbf{v})$. Let us state our principal results in this paper.

TheOREM 1.2. (i) For every vector $\mathbf{v}$ in $W_{1}$ and not in $D_{1}$, there exists a one-parameter family of classical solutions of the system $S(\mathbf{v})$. For each solution $(p, q)$ in the family, the transcendence degree of $\mathbf{C}(t, p, q)$ over $\mathbf{C}(t)$ equals one or zero. 
(ii) For every vector $\mathbf{v} \in D_{1}$, there exist two one-parameter families of classical solutions of the system $S(\mathbf{v})$. For each solution $(p, q)$ in the families, the transcendence degree of $\mathbf{C}(t, p, q)$ over $\mathbf{C}(t)$ equals one or zero.

(iii) For every $\mathbf{v} \in \mathbf{C}^{2}$, let $(p, q)$ be a transcendental solution of the system $S(\mathbf{v})$ different from those in (i) and (ii). Then neither the function $p$ nor the function $q$ is classical, and the transcendence degree of $\mathbf{C}(t, p, q)$ over $\mathbf{C}(t)$ equals two.

Remark 1.2. The statement (iii) implies the irreducibility of the third Painlevé equation (cf. [18]; see also Theorem 1.4.).

To prove Theorem 1.2, we may assume by the operation of $\mathbf{G}_{*}$ on $\Sigma$ that the vector $\mathbf{v}$ parametrizing the system $S(\mathbf{v})$ belongs to the fundamental region $\Gamma$ of the group $\mathbf{G}$. Therefore it is sufficient to prove the following:

THEOREM 1.3. (i) For every vector $\mathbf{v}_{1}=\left(v_{1}, v_{2}\right) \in \mathbf{C}^{2}$ such that $v_{1}-$ $v_{2}=0$, there exists a one-parameter family of classical solutions of $S\left(\mathbf{v}_{1}\right)$, which consists of solutions of the form $(1, q)$, where $q$ is a solution of a Riccati equation

$$
t \frac{d q}{d t}=q^{2}+v_{1} q+t
$$

(ii) For every $\mathbf{v}_{2}=\left(v_{1}, v_{2}\right) \in \mathbf{C}^{2}$ such that $v_{1}+v_{2}=0$, there exists a one-parameter family of classical solutions of $S\left(\mathbf{v}_{2}\right)$, which consists of solutions of the form $(0, q)$, where $q$ is a solution of a Riccati equation

$$
t \frac{d q}{d t}=-q^{2}+v_{1} q+t
$$

(iii) For every $\mathbf{v} \in \Gamma$, let $(p, q)$ be a transcendental solution of the system $S(\mathbf{v})$ different from those in (i) and (ii). Then neither the function $p$ nor the function $q$ is classical; the transcendence degree of $\mathbf{C}(t, p, q)$ over $\mathbf{C}(t)$ equals two.

The assertions (i) and (ii) are obvious. Therefore our main task is to prove the assertion (iii), which will be done in the following sections.

Using birational transformations in the group $\mathbf{G}_{*}$, we can explicitly write every classical solution in Theorem 1.2 by a classical solution in Theorem 1.3. In fact, let $(p, q)$ be a classical solution of $S(\mathbf{v})$ for a $\mathbf{v} \in W_{1}$. Since $W_{1} \cap \Gamma$ is a fundamental region of a $\mathbf{G}$-invariant subset $W_{1}$ of $\mathbf{C}^{2}$, there exist an element $g \in \mathbf{G}$ and a unique vector $\mathbf{v}_{0} \in W_{1} \cap \Gamma$ such that 
$\mathbf{v}=g\left(\mathbf{v}_{0}\right)$. Therefore, there exists a classical solution $\left(p_{0}, q_{0}\right)$ of $S\left(\mathbf{v}_{0}\right)$ in Theorem 1.3 such that $(p, q)=\gamma\left(p_{0}, q_{0}\right)$ for every $\gamma \in f^{-1}(g)$.

Now we introduce three variables $t^{\prime}, p^{\prime}, q^{\prime}$ by

$$
t^{\prime}=-t, \quad p^{\prime}=1-p, \quad q^{\prime}=-q .
$$

If we eliminate the letters $t, q, p$ from $S(\mathbf{v})\left(\mathbf{v}=\left(v_{1}, v_{2}\right)\right)$ and $(3)$, then we get the following system:

$$
\left\{\begin{array}{l}
t^{\prime} \frac{d q^{\prime}}{d t^{\prime}}=2\left(q^{\prime}\right)^{2} p^{\prime}-\left(q^{\prime}\right)^{2}+v_{1} q^{\prime}+t^{\prime} \\
t^{\prime} \frac{d p^{\prime}}{d t^{\prime}}=-2 q^{\prime}\left(p^{\prime}\right)^{2}+2 q^{\prime} p^{\prime}-v_{1} p^{\prime}+\frac{1}{2}\left(v_{1}-v_{2}\right)
\end{array}\right.
$$

This is nothing but the system $S\left(v_{1},-v_{2}\right)$. Therefore classical solutions of (i) and (ii) in Theorem 1.3 are exchanged each other by the transformation (3).

Let us now introduce a new variable $y$ by

$$
q=\frac{v_{1}}{2}+t \frac{d}{d t}(\log y)
$$

where $(d / d t)(\log y)=(d y / d t) / y$. If we substitute (4) into (2), then we have

$$
\frac{d^{2} y}{d t^{2}}+\frac{1}{t} \frac{d y}{d t}-\frac{1}{t^{2}}\left(t+\frac{v_{1}^{2}}{4}\right) y=0
$$

Moreover, setting

$$
t=-\frac{x^{2}}{4}
$$

and eliminating the variable $t$ from (5) and (6), we obtain the Bessel's differential equation

$$
\frac{d^{2} y}{d x^{2}}+\frac{1}{x} \frac{d y}{d x}+\left(1-\frac{v_{1}^{2}}{x^{2}}\right) y=0
$$

Therefore we see that the Riccati equations (1) and (2), and therefore all the transcendental classical solutions of $S(\mathbf{v})$ for $\mathbf{v} \in W_{1}$, are rationally generated from Bessel functions defined by (7).

Finally we state the result on the determination of transcendental classical solutions of the system $\tilde{S}(\mathbf{v})$ introduced in $\S 0$. We can construct a group of birational transformations of solutions of $\tilde{S}(\mathbf{v})$ associated with the 
group $\mathbf{G}$ similar to $S(\mathbf{v})$. Hence, by the same reason as the case of the system $S(\mathbf{v})$, we may assume $\mathbf{v} \in \Gamma$ to determine all the classical solutions of $\tilde{S}(\mathbf{v})$. Using the transformation (1) in $\S 0$, we can translate results for $\tilde{S}(\mathbf{v})$ into those for $S(\mathbf{v})$, and the converse is also possible. Therefore, from Theorem 1.3 we obtain the following result for the system $\tilde{S}(\mathbf{v})$ :

TheOREM 1.4. (i) For every $\mathbf{v}_{1}=\left(v_{1}, v_{2}\right) \in \mathbf{C}^{2}$ such that $v_{1}-v_{2}=0$, there exists a one-parameter family of classical solutions of $\tilde{S}\left(\mathbf{v}_{1}\right)$, which consists of solutions of the form $(T, Q)$, where $Q$ is a solution of a Riccati equation

$$
T \frac{d Q}{d T}=2 T Q^{2}+\left(2 v_{1}-1\right) Q+2 T
$$

(ii) For every $\mathbf{v}_{2}=\left(v_{1}, v_{2}\right) \in \mathbf{C}^{2}$ such that $v_{1}+v_{2}=0$, there exists a one-parameter family of classical solutions of $\tilde{S}\left(\mathbf{v}_{2}\right)$, which consists of solutions of the form $(0, Q)$, where $Q$ is a solution of a Riccati equation

$$
T \frac{d Q}{d T}=-2 T Q^{2}+\left(2 v_{1}-1\right) Q+2 T
$$

(iii) For every $\mathbf{v} \in \Gamma$, let $(P, Q)$ be a transcendental solution of the system $\tilde{S}(\mathbf{v})$ different from those in (i) and (ii). Then neither the function $P$ nor the function $Q$ is classical; the transcendence degree of $\mathbf{C}(T, P, Q)$ over $\mathbf{C}(T)$ equals two.

\section{$\S 2$. Necessary condition of the existence of invariant ideals}

Let $K$ be an ordinary differential overfield of the field $\mathbf{C}(t)$ of rational functions over $\mathbf{C}$, and let $K[p, q]$ be the polynomial ring over $K$ in two variables $p$ and $q$. We consider the following derivation $X(\mathbf{v})$ on $K[p, q]$ :

$X(\mathbf{v})=t \frac{\partial}{\partial t}+\left(2 q^{2} p-q^{2}+v_{1} q+t\right) \frac{\partial}{\partial q}+\left(-2 q p^{2}+2 q p-v_{1} p+\frac{v_{1}+v_{2}}{2}\right) \frac{\partial}{\partial p}$

From now on we fix the vector $\mathbf{v} \in \mathbf{C}^{2}$. In [23], §1, Umemura introduced the condition $(\mathrm{J})$ for $X(\mathbf{v})$ as follows:

(J) For any ordinary differential field extension $K / \mathbf{C}(t)$, there exists no principal ideal $I$ of $K[p, q]$ such that $0 \subsetneq I \subsetneq K[p, q]$ and $X(\mathbf{v}) I \subset I$.

Let us show the following proposition whose proof is the main part of the proof of Theorem 1.3. 
Proposition 2.1. If the derivation $X(\mathbf{v})$ does not satisfy the condition $(\mathrm{J})$ for a vector $\mathbf{v}=\left(v_{1}, v_{2}\right) \in \mathbf{C}^{2}$, then there exist non-negative integers $h, i, j$ such that

$$
i+j \geq 1
$$

and

$$
i\left(v_{1}+v_{2}\right)+j\left(v_{1}-v_{2}\right)+2 h\left(1-v_{1}\right)=0
$$

Proof. We shall proceed in eight steps.

Step 1. By hypothesis there exists a differential overfield $K$ of $\mathbf{C}(t)$ such that there exists an $X(\mathbf{v})$-invariant principal ideal $I$ properly between the zero-ideal and $K[p, q]$. Let $F \in K[p, q]$ be a generator of $I$. Then we have $I=(F), F \notin K$ and

$$
X(\mathbf{v}) F=G F
$$

for some $G \in K[p, q]$.

To investigate the equation (3), we introduce the following two gradings in the polynomial ring $K[p, q]$.

In the first grading we define the weights of $p$ and $q$ to be 0 and 1 respectively so that the weight of a monomial $a p^{i} q^{j}(0 \neq a \in K)$ in $K[p, q]$ is $j$. Let $R_{d}$ be the $K$-linear subspace of $K[p, q]$ generated over $K$ by all the monomials of weight $d$. So we have $R_{d}=K[p] \cdot q^{d}$ for every integer $d \geq 0$, and $K[p, q]$ becomes a graded ring: $K[p, q]=\bigoplus_{d \geq 0} R_{d}, R_{d} \cdot R_{d^{\prime}} \subseteq R_{d+d^{\prime}}$. We define three derivations $X_{i}$ 's $(i=-1,0,1)$ by

$$
\begin{aligned}
X_{1} & =(2 p-1) q^{2} \frac{\partial}{\partial q}-2 q(p-1) p \frac{\partial}{\partial p} \\
X_{0} & =t \frac{\partial}{\partial t}+v_{1} q \frac{\partial}{\partial q}+\left(-v_{1} p+\frac{v_{1}+v_{2}}{2}\right) \frac{\partial}{\partial p} \\
X_{-1} & =t \frac{\partial}{\partial q}
\end{aligned}
$$

so that we have $X(\mathbf{v})=X_{1}+X_{0}+X_{-1}$ and each $X_{i}$ maps $R_{d}$ to $R_{d+i}$.

In the second grading we define the weights of $p$ and $q$ to be 2 and -1 respectively so that the weight of a monomial $a p^{i} q^{j}(0 \neq a \in K)$ in $K[p, q]$ is $2 i-j$. Let $R_{d}^{\prime}$ be the $K$-linear subspace of $K[p, q]$ generated over $K$ by all the monomials of weight $d$. We have $R_{-d}^{\prime}=K\left[p q^{2}\right] \cdot q^{d}$, $R_{2 d-1}^{\prime}=K\left[p q^{2}\right] \cdot p^{d} q, R_{2 d}^{\prime}=K\left[p q^{2}\right] \cdot p^{d}$ for every integer $d \geq 0$. This defines another grading in $K[p, q]: K[p, q]=\oplus_{-\infty<d<\infty} R_{d}^{\prime}, R_{d}^{\prime} \cdot R_{d^{\prime}}^{\prime} \subseteq R_{d+d^{\prime \prime}}^{\prime}$. We 
define four derivations $X_{i}^{\prime \prime s}(i=-2,-1,0,1)$ by

$$
\begin{aligned}
X_{1}^{\prime} & =\left(2 p q^{2}+t\right) \frac{\partial}{\partial q}-2 p^{2} q \frac{\partial}{\partial p} \\
X_{0}^{\prime} & =t \frac{\partial}{\partial t}+v_{1} q \frac{\partial}{\partial q}-v_{1} p \frac{\partial}{\partial p} \\
X_{-1}^{\prime} & =-q^{2} \frac{\partial}{\partial q}+2 p q \frac{\partial}{\partial p} \\
X_{-2}^{\prime} & =\frac{v_{1}+v_{2}}{2} \frac{\partial}{\partial p}
\end{aligned}
$$

so that we have $X(\mathbf{v})=X_{1}^{\prime}+X_{0}^{\prime}+X_{-1}^{\prime}+X_{-2}^{\prime}$ and each $X_{i}^{\prime}$ maps $R_{d}^{\prime}$ to $R_{d+i}^{\prime}$.

Let us determine the form of the polynomial $G$ in (3). Since the highest part $X_{1}$ of $X(\mathbf{v})$ is of weight one with respect to the first grading, the polynomial $G$ belongs to the direct sum $R_{0} \oplus R_{1}$. Namely we have $G=$ $g_{1} q+g$ for some $g_{1}, g \in R_{0}$. In addition, since the highest part $X_{1}^{\prime}$ of $X(\mathbf{v})$ is also of weight one with respect to the second grading, the polynomial $G$ belongs to the direct sum $\oplus_{d \leq 1} R_{d}^{\prime}$. Therefore we have $g \in K$ and $g_{1}=\lambda p+\mu$ for some $\lambda, \mu \in K$. Namely we have

$$
G=(\lambda p+\mu) q+g
$$

for some $\lambda, \mu, g \in K$.

Let us decompose the polynomial $F$ with respect to the first grading of $K[p, q]$. Then there exist a non-negative integer $m \geq 0$ and a unique collection of $m+1$ homogeneous polynomials $F_{d} \in R_{d}(0 \leq d \leq m)$ such that $F=F_{0}+\cdots+F_{m}, F_{m} \neq 0$ and, if $m=0, F_{0} \notin K$. Hence the equation (3) is written as

$$
\left(X_{1}+X_{0}+X_{-1}\right)\left(F_{m}+\cdots+F_{0}\right)=\{(\lambda p+\mu) q+g\}\left(F_{m}+\cdots+F_{0}\right) .
$$

Comparing the homogeneous parts of both sides of (5), we have a system of $m+3$ equations equivalent to (3):

$$
X_{1} F_{d}=(\lambda p+\mu) q F_{d}+g F_{d+1}-X_{0} F_{d+1}-X_{-1} F_{d+2}
$$

for each integer $d$ such that $-2 \leq d \leq m$. Here we consider $F_{-2}=F_{-1}=$ $F_{m+1}=F_{m+2}=0$.

Let us decompose the polynomial $F$ with respect to the second grading of $K[p, q]$. Then there exist two integers $n, n^{\prime}\left(n^{\prime} \leq n\right)$ and a unique 
collection of $n-n^{\prime}+1$ homogeneous polynomials $F_{d}^{\prime} \in R_{d}^{\prime}\left(n^{\prime} \leq d \leq n\right)$ such that $F=F_{n^{\prime}}^{\prime}+\cdots+F_{n}^{\prime}, F_{n^{\prime}}^{\prime} F_{n}^{\prime} \neq 0$ and, if $n=n^{\prime}=0, F_{0}^{\prime} \notin K$. Hence the equation (3) is written as

$$
\begin{aligned}
\left(X_{1}^{\prime}+X_{0}^{\prime}+X_{-1}^{\prime}+X_{-2}^{\prime}\right) & \left(F_{n^{\prime}}^{\prime}+\cdots+F_{n}^{\prime}\right) \\
= & (\lambda p q+\mu q+g)\left(F_{n^{\prime}}^{\prime}+\cdots+F_{n}^{\prime}\right) .
\end{aligned}
$$

Comparing the homogeneous parts of both sides of (7), we have a system of $n-n^{\prime}+4$ equations equivalent to (3):

(8) ${ }_{d} X_{1}^{\prime} F_{d}^{\prime}=\lambda p q F_{d}^{\prime}+g F_{d+1}^{\prime}+\mu g F_{d+2}^{\prime}-X_{0}^{\prime} F_{d+1}^{\prime}-X_{-1}^{\prime} F_{d+2}^{\prime}-X_{-2}^{\prime} F_{d+3}^{\prime}$

for each integer $d$ such that $n^{\prime}-3 \leq d \leq n$. Here we consider $F_{n^{\prime}-3}^{\prime}=$ $F_{n^{\prime}-2}^{\prime}=F_{n^{\prime}-1}^{\prime}=F_{n+1}^{\prime}=F_{n+2}^{\prime}=F_{n+3}^{\prime}=0$.

Remark 2.1. By the same argument as in Subsection 2.5 in [23], we see that the gradings above come from the Newton polygon of the derivation $X(\mathbf{v})$, which is represented by the following picture:

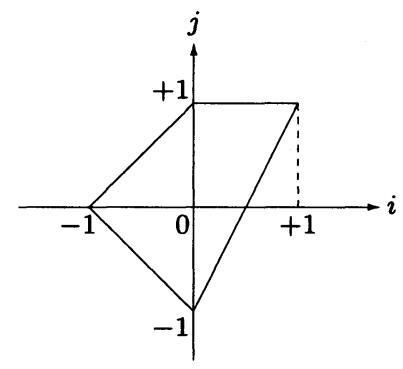

Here an integral point $(i, j) \neq(0,0)$ in $\mathbf{R}^{2}$ represents the derivation in $X(\mathbf{v})$ of the form $u p^{i+1} q^{j} \partial / \partial p+v p^{i} q^{j+1} \partial / \partial q(u, v \in K)$; the point $(0,0)$ represents that of the form $t \partial / \partial t+u p \partial / \partial p+v q \partial / \partial q(u, v \in K)$.

Step 2. We prepare three lemmas (Lemmas 2.2-2.4) to investigate the equations $(6)_{d}$ and $(8)_{d}$.

LEMMA 2.2. Let $d$ be a non-negative integer and $k$ be a positive integer. Let $A$ be a polynomial in $R_{d}$, and let $\lambda^{\prime}$ and $\mu^{\prime}$ be elements of $K$. If $\mu^{\prime}+$ $d-2 l+2 \neq 0$ for every integer $l$ such that $1 \leq l \leq k$ and if $A$ satisfies a congruence

$$
X_{1} A \equiv\left(\lambda^{\prime} p+\mu^{\prime}\right) q A \quad\left(\bmod p^{k}\right),
$$

then $A \equiv 0 \quad\left(\bmod p^{k}\right)$. 
Lemma 2.3. Let $d, k, A, \lambda^{\prime}, \mu^{\prime}$ be as above. If $\lambda^{\prime}+\mu^{\prime}-d+2 l-2 \neq 0$ for every integer $l$ such that $1 \leq l \leq k$ and if $A$ satisfies a congruence

$$
X_{1} A \equiv\left(\lambda^{\prime} p+\mu^{\prime}\right) q A \quad\left(\bmod (p-1)^{k}\right),
$$

then $A \equiv 0 \quad\left(\bmod (p-1)^{k}\right)$.

Proof of Lemma 2.2. We denote by $K[T]$ the polynomial ring in one variable $T$ over $K$. Let $\varphi$ be the $K$-algebra morphism of $K[p, q]$ onto $K[T]$ defined by $\varphi(q)=T$ and $\varphi(p)=0$. The kernel Ker $\varphi$ is the principal ideal generated by $p$. Then the following diagram (11) is commutative:

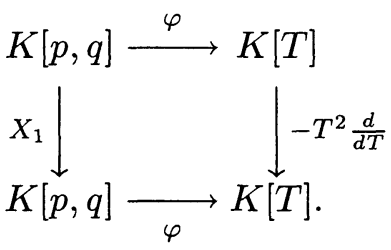

Hence the kernel $\operatorname{Ker} \varphi=(p)$ is $X_{1}$-invariant. In fact we have a formula

$$
X_{1}(p)=-2 q(p-1) p .
$$

Let us show $A \equiv 0 \quad\left(\bmod p^{l}\right)$ by induction on $l(1 \leq l \leq k)$. Since $A$ is in $R_{d}$, there exists an element $B \in R_{0}=K[p]$ such that $A=B \cdot q^{d}$. If we apply $\varphi$ to both sides of (9), we have

$$
\varphi\left(X_{1} A\right)=\varphi\left(\lambda^{\prime} p+\mu^{\prime}\right) \varphi(q A) .
$$

This is equivalent to

$$
-T^{2} \frac{d}{d T} \varphi(A)=\varphi\left(\lambda^{\prime} p+\mu^{\prime}\right) \varphi(q A)
$$

by the commutative diagram (11). Since $\varphi(A)=\varphi(B) T^{d}$, it follows that

$$
\left(\mu^{\prime}+d\right) \varphi(B) T^{d+1}=0 .
$$

Since $\mu^{\prime}+d \neq 0$ by hypothesis, we have $\varphi(B)=0$ and hence $A \equiv 0$ $(\bmod p)$. This proves the case $l=1$. Assume that $A \equiv 0\left(\bmod p^{l-1}\right)$ for $l \geq 2$. We show $A \equiv 0 \quad\left(\bmod p^{l}\right)$. By assumption there exists an element $C \in R_{0}$ such that

$$
A=C p^{l-1} q^{d} .
$$


If we substitute (13) into (9) and divide both sides of the resulting congruence by $p^{l-1}$, then we get

(14) $X_{1}\left(C q^{d}\right) \equiv\left\{\left(\lambda^{\prime}+2 l-2\right) p+\mu^{\prime}-2 l+2\right\} C q^{d+1} \quad\left(\bmod p^{k-l+1}\right)$.

If we apply $\varphi$ to (14), we have an equality

$$
\left(\mu^{\prime}+d-2 l+2\right) \varphi(C) T^{d+1}=0 .
$$

Since $\mu^{\prime}+d-2 l+2 \neq 0$ by hypothesis, we have $\varphi(C)=0$ and hence $A \equiv 0$ $\left(\bmod p^{l}\right)$. Thus Lemma 2.2 is proved.

Proof of Lemma 2.3. Let $\psi$ be the $K$-algebra morphism of $K[p, q]$ onto $K[T]$ defined by $\psi(q)=T$ and $\psi(p)=1$. The kernel Ker $\psi$ is the principal ideal generated by $p-1$. Then the following diagram (15) is commutative:

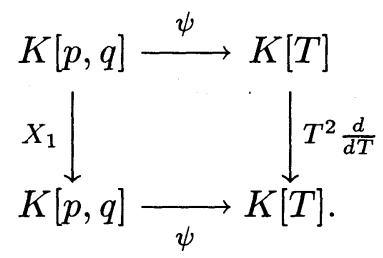

Hence the kernel $\operatorname{Ker} \psi=(p-1)$ is $X_{1}$-invariant. In fact we have a formula

$$
X_{1}(p-1)=-2 q p(p-1)
$$

We can show $A \equiv 0 \quad\left(\bmod (p-1)^{l}\right)$ by induction on $l(1 \leq l \leq k)$ in the same procedure as in the proof of Lemma 2.2 if we use $\psi$ and (15) for $\varphi$ and (11). The detail is left to the reader.

Remark 2.2. The commutative diagrams (11) and (15) are obtained in the following procedure (cf. [23], [25]). Let us determine homogeneous $K$-algebra morphisms $\theta$ such that the following diagram is commutative:

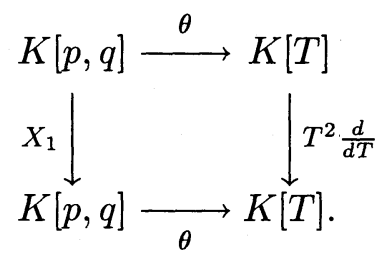


Here we consider the polynomial ring $K[T]$ as a graded ring in the usual way. Hence, if we set $\theta(p)=a, \theta(q)=b T$ with $a, b \in K$, then we get a system of algebraic equations:

$$
\left\{\begin{array}{c}
(a-1) a b=0, \\
(2 a-1) b^{2}=b .
\end{array}\right.
$$

Therefore we have the solutions $(a, b)=(0,-1),(1,1),(a, 0)$. The first two of them define the expected morphisms $\varphi$ and $\psi$ respectively, and the remainder has no importance.

LeMma 2.4. Let $d$ be an integer and let $k$ be a positive integer. Let $A$ be a polynomial in $R_{d}^{\prime}$, and let $\lambda^{\prime}$ be an element of $K$. If $\lambda^{\prime}+d-2 l+2 \neq 0$ for every integer $l$ such that $1 \leq l \leq k$ and if $A$ satisfies a congruence

$$
X_{1}^{\prime} A \equiv \lambda^{\prime} p q A \quad\left(\bmod \left(p q^{2}+t\right)^{k}\right)
$$

then $A \equiv 0 \quad\left(\bmod \left(p q^{2}+t\right)^{k}\right)$.

Proof. We denote by $K\left[T, T^{-1}\right]$ the ring of Laurent polynomials in one variable $T$ over $K$. Let $\Phi$ be the $K$-algebra morphism of $K[p, q]$ onto $K\left[T, T^{-1}\right]$ defined by $\Phi(p)=-t^{-1} T^{2}$ and $\Phi(q)=t T^{-1}$. The kernel Ker $\Phi$ is the principal ideal generated by $p q^{2}+t$. Then the following diagram (18) is commutative:

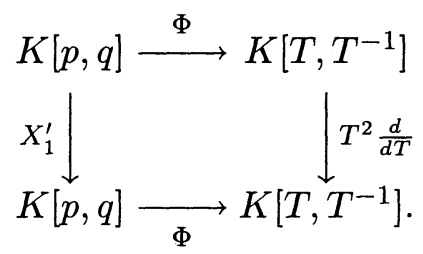

Hence the kernel $\operatorname{Ker} \Phi=\left(p q^{2}+t\right)$ is $X_{1}^{\prime}$-invariant. In fact we have a formula

$$
X_{1}^{\prime}\left(p q^{2}+t\right)=2 p q\left(p q^{2}+t\right) .
$$

Let us now show $A \equiv 0\left(\bmod \left(p q^{2}+t\right)^{l}\right)$ by induction on $l(1 \leq l \leq k)$. The proof is divided into the following three cases: (i) the case where the integer $d$ is non-negative and even; (ii) the case where it is non-negative and odd; (iii) the case where it is negative.

(i) Suppose that the integer $d$ is non-negative and even. Then there exists an element $B \in R_{0}^{\prime}=K\left[p q^{2}\right]$ such that $A=B p^{\frac{d}{2}}$. If we apply $\Phi$ to both sides of (17), then we have

$$
\Phi\left(X_{1}^{\prime} A\right)=\Phi\left(\lambda^{\prime} p q A\right)
$$


This is equivalent to

$$
T^{2} \frac{d}{d T} \Phi(A)=\lambda^{\prime} \Phi(p q) \Phi(A)
$$

by the commutative diagram (18). Since $\Phi(A)=\Phi(B)(-t)^{-\frac{d}{2}} T^{d}$, it follows that

$$
\left(\lambda^{\prime}+d\right) \Phi(B)=0 \text {. }
$$

Since $\lambda^{\prime}+d \neq 0$ by hypothesis, we have $\Phi(B)=0$ and hence $A \equiv 0$ $\left(\bmod p q^{2}+t\right)$. This proves the case $l=1$. Assume that $A \equiv 0 \quad\left(\bmod \left(p q^{2}+\right.\right.$ $\left.t)^{l-1}\right)$ for $l \geq 2$. We show $A \equiv 0 \quad\left(\bmod \left(p q^{2}+t\right)^{l}\right)$. By assumption there exists a $C \in R_{0}^{\prime}$ such that

$$
A=C\left(p q^{2}+t\right)^{l-1} p^{\frac{d}{2}} .
$$

If we substitute (20) into (17) and divide both sides of the resulting congruence by $\left(p q^{2}+t\right)^{l-1}$, then we get

$$
X_{1}^{\prime}\left(C p^{\frac{d}{2}}\right) \equiv\left(\lambda^{\prime}-2 l+2\right) p q C p^{\frac{d}{2}}\left(\bmod \left(p q^{2}+t\right)^{k-l+1}\right) .
$$

If we apply $\Phi$ to (21), we have an equality

$$
\left(\lambda^{\prime}+d-2 l+2\right) \Phi(C)=0 .
$$

Since $\lambda^{\prime}+d-2 l+2 \neq 0$ by hypothesis, we have $\Phi(C)=0$ and hence $A \equiv 0$ $\left(\bmod \left(p q^{2}+t\right)^{l}\right)$.

(ii) Suppose that the integer $d$ is non-negative and odd. Then there exists an element $B \in R_{0}^{\prime}$ such that $A=B p^{\frac{d+1}{2}} q$. The successive argument is quite similar to the argument developed in the case (i). So the detail is left to the reader.

(iii) Suppose that the integer $d$ is negative. Then there exists an element $B \in R_{0}^{\prime}$ such that $A=B q^{-d}$. The succesive argument is quite similar to the argument developed in the case (i). So the detail is left to the reader. Thus Lemma 2.4 is proved.

Remark 2.3. The commutative diagram (18) is obtained in the following procedure (cf. [23], [25]; see also Remark 2.2). Let us determine the homogeneous $K$-algebra morphism $\theta$ such that the following diagram is commutative:

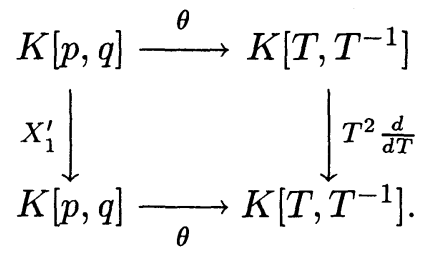


Here we consider the ring $K\left[T, T^{-1}\right]$ of Laurent polynomials as a graded ring in the usual way. Hence, if we set $\theta(p)=a T^{2}, \theta(q)=b T^{-1}$ with $a, b \in K$, then we get a system of algebraic equations:

$$
\left\{\begin{aligned}
-2 a^{2} b & =2 a \\
2 a b^{2}+t & =-b
\end{aligned}\right.
$$

Therefore we have the solutions $(a, b)=\left(-t^{-1}, t\right),(0,-t)$. The former gives the expected morphism $\Phi$, and the latter has no importance.

Step 3. Now we come back to the proof of the proposition. The polynomial $F_{m}$ satisfies the equation $(6)_{m}$ :

$$
X_{1} F_{m}=(\lambda p+\mu) q F_{m}
$$

We claim that $\frac{1}{2}(m+\mu)$ is a non-negative integer. Otherwise, we would have $\mu+m-2 l+2 \neq 0$ for every integer $l \geq 1$. By Lemma 2.2 we would have $F_{m} \equiv 0 \quad\left(\bmod p^{k}\right)$ for every integer $k \geq 1$. Hence we would have $F_{m}=0$, and this is a contradiction. Similarly we see by Lemma 2.3 that $\frac{1}{2}(m-\lambda-\mu)$ is a non-negative integer. If we set $i=\frac{1}{2}(m+\mu)$ and $j=\frac{1}{2}(m-\lambda-\mu)$, then we have

$$
\lambda=2 m-2 i-2 j
$$

and

$$
\mu=2 i-m \text {. }
$$

If $i \geq 1$, we have $F_{m} \equiv 0 \quad\left(\bmod p^{i}\right)$ by Lemma 2.2 because $\mu+m-2 l+2 \neq$ 0 for every integer $l$ such that $1 \leq l \leq i$. If $j \geq 1$, we have $F_{m} \equiv 0$ $\left(\bmod (p-1)^{j}\right)$ by Lemma 2.3 because $\lambda+\mu-m+2 l-2 \neq 0$ for every integer $l$ such that $1 \leq l \leq j$. Hence, there exists a non-zero element $c \in R_{0}=K[p]$ such that

$$
F_{m}=c p^{i}(p-1)^{j} q^{m}
$$

where we allow $i=0$ or $j=0$. If we substitute (24) into $(6)_{m}$, we have by (22) and (23) an equation for $c: X_{1} c=0$. Since $c$ is a polynomial in $p$ over $K$, we have $c \in K$ immediately.

Step 4 . The polynomial $F_{n}^{\prime}$ satisfies the equation $(8)_{n}$ :

$$
X_{1}^{\prime} F_{n}^{\prime}=\lambda p q F_{n}^{\prime}
$$


Firstly we claim that the polynomial $F_{n}^{\prime}$ is not divisible by $q$. Otherwise, there would exist an integer $k \geq 1$ and a polynomial $A$ in $R_{n+k}^{\prime}$ such that

$$
F_{n}^{\prime}=A q^{k}
$$

and

$$
q \nmid A .
$$

If we substitute $(25)$ into $(8)_{n}$, we have an equation for $A$ :

$$
\left(X_{1}^{\prime} A\right) q^{k}+k q^{k-1}\left(X_{1}^{\prime} q\right) A=\lambda p q A q^{k} .
$$

Since $q \nmid X_{1}^{\prime} q$, we have $q \mid A$. This contradicts (26). Therefore we see that the integer $n$ is non-negative and even. Secondly we claim that $\frac{1}{2}(\lambda+n)$ is a non-negative integer. Otherwise, we would have $\lambda+n-2 l+2 \neq 0$ for every integer $l \geq 1$. By Lemma 2.4 we would have $F_{n}^{\prime} \equiv 0\left(\bmod \left(p q^{2}+t\right)^{k}\right)$ for every integer $k \geq 1$. Hence we would have $F_{n}^{\prime}=0$ and this is a contradiction. Setting $h=\frac{1}{2}(\lambda+n)$, we have

$$
\lambda=2 h-n
$$

If $h \geq 1$, we have $F_{n}^{\prime} \equiv 0 \quad\left(\bmod \left(p q^{2}+t\right)^{h}\right)$ by Lemma 2.4 because $\lambda+n-$ $2 l+2 \neq 0$ for every integer $l$ such that $1 \leq l \leq h$. Hence, there exists a non-zero element $c^{\prime} \in R_{0}^{\prime}=K\left[p q^{2}\right]$ such that

$$
F_{n}^{\prime}=c^{\prime}\left(p q^{2}+t\right)^{h} p^{\frac{n}{2}}
$$

where we allow $h=0$. If we substitute $(28)$ into $(8)_{n}$, we have an equation for $c^{\prime}: X_{1}^{\prime} c^{\prime}=0$. Since $c^{\prime}$ is a polynomial in $p q^{2}$ over $K$, we have $c^{\prime} \in K$ immediately.

Step 5. By the same argument as in [23], Subsection 2.5, we find the following figure of the Newton polygon of the invariant polynomial $F$ :

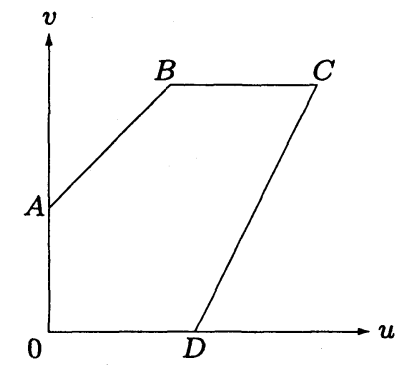


Here an integral point $(u, v)$ in $\mathbf{R}^{2}$ represents a monomial $\gamma p^{u} q^{v}(\gamma \in K)$. The side $B C$ represents the polynomial $F_{m}$; the side $C D$ represents the polynomial $F_{n}^{\prime}$. By the same argument as when the polynomials $F_{m}$ and $F_{n}^{\prime}$ were obtained, we find that the side $A B$ represents the polynomial $(-1)^{j} c\left(p q+\frac{v_{1}+v_{2}}{2}\right)^{i} q^{m-i}$. Therefore the Cartesian coordinates of the vertices $O, A, B, C, D$ are $(0,0),(0, m-i),(i, m),(i+j, m)=\left(h+\frac{n}{2}, 2 h\right)$, $\left(\frac{n}{2}, 0\right)$. Namely we have the following equalities:

$$
\begin{aligned}
i+j & =h+\frac{n}{2} ; \\
m & =2 h ; \\
c & =c^{\prime} .
\end{aligned}
$$

In particular we see from (29) and (30) that (22) and (27) are compatible each other. The polynomial $c^{-1} F$ is $X(\mathbf{v})$-invariant and generates the ideal $I=(F)$ introduced in Step 1. Hence we may assume $c=1$. Namely we have

$$
\begin{aligned}
F_{m} & =p^{i}(p-1)^{j} q^{m}, \\
F_{n}^{\prime} & =\left(p q^{2}+t\right)^{h} p^{\frac{n}{2}}
\end{aligned}
$$

from (24), (28), (31). If $m=0$, we have $F=F_{0}=p^{i}(p-1)^{j}$. Since $F \notin K$, we have $i+j \geq 1$. If $m \geq 1$, we have $i+j \geq h \geq 1$ by (29) and (30). Therefore we have (1) as required.

Step 6 . The polynomial $F_{m-1}$ satisfies the equation $(6)_{m-1}$ :

$(6)_{m-1}$

$$
X_{1} F_{m-1}=(\lambda p+\mu) q F_{m-1}+g F_{m}-X_{0} F_{m} .
$$

If we substitute (32) into $(6)_{m-1}$, we get

$$
\begin{aligned}
X_{1} F_{m-1}= & (\lambda p+\mu) q F_{m-1} \\
& +\left(g-m v_{1}+i v_{1}+j v_{1}\right) p^{i}(p-1)^{j} q^{m} \\
& -i \frac{v_{1}+v_{2}}{2} p^{i-1}(p-1)^{j} q^{m} \\
& +j \frac{v_{1}-v_{2}}{2} p^{i}(p-1)^{j-1} q^{m}
\end{aligned}
$$

where $\lambda$ and $\mu$ are given by (22) and (23). If $m=0$, we have $h=0$ by (30). Then the equality (34) is turned into

$$
\left(g+i v_{1}+j v_{1}\right) p(p-1)-i \frac{v_{1}+v_{2}}{2}(p-1)+j \frac{v_{1}-v_{2}}{2} p=0 .
$$


Since $p(p-1), p-1$ and $p$ are linearly independent over $K$, we have

$$
g=-(i+j) v_{1}
$$

and

$$
i\left(v_{1}+v_{2}\right)=j\left(v_{1}-v_{2}\right)=0 .
$$

The equality (37) with $h=0$ satisfies (2). Therefore the proposition is proved in the case $m=0$. Assume $m \geq 1$. Since $X_{1}$ is a derivation, we have

$$
X_{1}\left(p(p-1) F_{m-1}\right)=-2(2 p-1) q p(p-1) F_{m-1}+p(p-1) X_{1} F_{m-1} .
$$

Eliminating $X_{1} F_{m-1}$ from (34) and (38), we have

$$
\begin{aligned}
X_{1}\left(p(p-1) F_{m-1}\right)= & \{(\lambda-4) p+\mu+2\} q p(p-1) F_{m-1} \\
& +\left(g-m v_{1}+i v_{1}+j v_{1}\right) p^{i+1}(p-1)^{j+1} q^{m} \\
& -i \frac{v_{1}+v_{2}}{2} p^{i}(p-1)^{j+1} q^{m} \\
& +j \frac{v_{1}-v_{2}}{2} p^{i+1}(p-1)^{j} q^{m} .
\end{aligned}
$$

We have $X_{1}\left(p(p-1) F_{m-1}\right) \equiv\{(\lambda-4) p+\mu+2\} q p(p-1) F_{m-1} \quad\left(\bmod p^{i}\right)$. If $i \geq 1$, we have $p(p-1) F_{m-1} \equiv 0 \quad\left(\bmod p^{i}\right)$ by Lemma 2.2 because $(\mu+2)+(m-1)-2 l+2=2 i-2 l+3 \neq 0$ for every integer $l$ such that $1 \leq l \leq i$. We also have $X_{1}\left(p(p-1) F_{m-1}\right) \equiv\{(\lambda-4) p+\mu+2\} q p(p-1) F_{m-1}$ $\left(\bmod (p-1)^{j}\right)$. If $j \geq 1$, we have $p(p-1) F_{m-1} \equiv 0\left(\bmod (p-1)^{j}\right)$ by Lemma 2.3 because $(\lambda-4)+(\mu+2)-(m-1)+2 l-2=-2 j+2 l-3 \neq 0$ for every integer $l$ such that $1 \leq l \leq j$. We have $p(p-1) F_{m-1} \equiv 0$ $\left(\bmod p^{i}(p-1)^{j}\right)$ by $(1)$. Therefore there exists an element $B \in R_{0}=K[p]$ such that

$$
p(p-1) F_{m-1}=B p^{i}(p-1)^{j} q^{m-1} .
$$

If we substitute (40) into (39) and divide both sides of the resulting equation by $p^{i}(p-1)^{j} q^{m-1}$, then we obtain an equation for $B$

$$
\begin{aligned}
L(B)= & \left(g-m v_{1}+i v_{1}+j v_{1}\right) p(p-1) q \\
& -i \frac{v_{1}+v_{2}}{2}(p-1) q \\
& +j \frac{v_{1}-v_{2}}{2} p q,
\end{aligned}
$$

where we put $L(B)=X_{1} B+(2 p-1) q B$. $L$ defines a $K$-linear mapping of $R_{0}$ into $R_{1}$. Let $V_{0}$ be the $K$-linear subspace of $R_{0}$ generated by $p$ and $p-1$, 
and let $V_{1}$ be the $K$-linear subspace of $R_{1}$ generated by $p q$ and $(p-1) q$. If we consider the following formulae

$$
\begin{aligned}
L(p) & =p q \\
L(p-1) & =-(p-1) q,
\end{aligned}
$$

then we see that the restriction of $L$ to $V_{0}$ induces a $K$-linear isomorphism of $V_{0}$ onto $V_{1}$. Furthermore, if $A$ is a polynomial in $R_{0}$ of degree $d \geq 2$ in $p$, then $L(A)$ is a polynomial in $R_{1}$ of degree $d+1$ in $p$. Therefore it follows that the polynomial $B$ is of degree at most one in $p$, and that the vector $p(p-1) q$ does not belong to the image of $R_{0}$ by $L: p(p-1) q \notin L\left(R_{0}\right)$. If we set

$$
B=x p+y(p-1)
$$

with $x, y \in K$ and substitute it into (41), then we obtain

$$
\begin{aligned}
& x=j \frac{v_{1}-v_{2}}{2}, \\
& y=i \frac{v_{1}+v_{2}}{2}, \\
& g=(m-i-j) v_{1},
\end{aligned}
$$

so that

$$
B=j \frac{v_{1}-v_{2}}{2} p+i \frac{v_{1}+v_{2}}{2}(p-1) .
$$

From (40), (48), we obtain

(49) $F_{m-1}=j \frac{v_{1}-v_{2}}{2} p^{i}(p-1)^{j-1} q^{m-1}+i \frac{v_{1}+v_{2}}{2} p^{i-1}(p-1)^{j} q^{m-1}$.

Step 7 . The polynomial $F_{n-1}^{\prime}$ satisfies the equation $(8)_{n-1}$ :

$(8)_{n-1}$

$$
X_{1}^{\prime} F_{n-1}^{\prime}=\lambda p q F_{n-1}^{\prime}+g F_{n}^{\prime}-X_{0}^{\prime} F_{n}^{\prime}
$$

If we substitute (33) into $(8)_{n-1}$, we get

$$
\begin{aligned}
X_{1}^{\prime} F_{n-1}= & \lambda p q F_{n-1}^{\prime} \\
& +\left(g-h v_{1}+\frac{n}{2} v_{1}\right)\left(p q^{2}+t\right)^{h} p^{\frac{n}{2}} \\
& +\left(v_{1}-1\right) h t\left(p q^{2}+t\right)^{h-1} p^{\frac{n}{2}}
\end{aligned}
$$


where $\lambda$ is given by (27). If $h=0$, we have $m=0$ by (30). As was seen in Step 6 , the proposition is proved in this case. We assume $h \geq 1$. Since $X_{1}^{\prime}$ is a derivation, we have

$$
X_{1}^{\prime}\left(\left(p q^{2}+t\right) F_{n-1}^{\prime}\right)=2 p q\left(p q^{2}+t\right) F_{n-1}^{\prime}+\left(p q^{2}+t\right) X_{1}^{\prime} F_{n-1}^{\prime} .
$$

Eliminating $X_{1}^{\prime} F_{n-1}^{\prime}$ from (50) and (51), we have

$$
\begin{aligned}
X_{1}^{\prime}\left(\left(p q^{2}+t\right) F_{n-1}^{\prime}\right)= & (\lambda+2) p q\left(p q^{2}+t\right) F_{n-1}^{\prime} \\
& +\left(g-h v_{1}+\frac{n}{2} v_{1}\right)\left(p q^{2}+t\right)^{h+1} p^{\frac{n}{2}} \\
& +\left(v_{1}-1\right) h t\left(p q^{2}+t\right)^{h} p^{\frac{n}{2}}
\end{aligned}
$$

We have $X_{1}^{\prime}\left(\left(p q^{2}+t\right) F_{n-1}^{\prime}\right) \equiv(\lambda+2) p q\left(p q^{2}+t\right) F_{n-1}^{\prime}\left(\bmod \left(p q^{2}+t\right)^{h}\right)$. Since $(\lambda+2)+(n-1)-2 l+2=2 h+3-2 l \neq 0$ for every integer $l$ such that $1 \leq l \leq h$, we have $\left(p q^{2}+t\right) F_{n-1}^{\prime} \equiv 0 \quad\left(\bmod \left(p q^{2}+t\right)^{h}\right)$ by Lemma 2.4 . Then there exists an element $C \in R_{0}^{\prime}$ such that

$$
\left(p q^{2}+t\right) F_{n-1}^{\prime}=C\left(p q^{2}+t\right)^{h} p^{\frac{n}{2}} q .
$$

If we substitute (53) into (52) and divide both sides of the resulting equation by $\left(p q^{2}+t\right)^{h} p^{\frac{n}{2}}$, then we obtain an equation for $C$ :

$$
\left.L^{\prime}(C)=\left(g-h v_{1}+\frac{n}{2} v_{1}\right) p q^{2}\right]+\left(g-h+\frac{n}{2} v_{1}\right) t
$$

where we put $L^{\prime}(C)=q X_{1}^{\prime} C+t C$. $L^{\prime}$ defines a $K$-linear endomorphism of $R_{0}^{\prime}$. Since $L^{\prime}(1)=t$ and $p q^{2} \notin L^{\prime}\left(R_{0}^{\prime}\right)$, we get

$$
\begin{aligned}
C & =g-h+\frac{n}{2} v_{1}, \\
g & =h v_{1}-\frac{n}{2} v_{1}
\end{aligned}
$$

from (54). Therefore we obtain

$$
F_{n-1}^{\prime}=h\left(v_{1}-1\right)\left(p q^{2}+t\right)^{h-1} p^{\frac{n}{2}} q
$$

from (53), (55), (56). Finally we notice that (47) and (56) are compatible each other by (29) and (30).

Step 8. From (49) and (57), the coefficient of the monomial $p^{i+j-1} q^{m-1}$ $=p^{h+\frac{n}{2}-1} q^{2 h-1}$ in $F$ is represented in two ways. Namely the coefficient of $p^{i+j-1} q^{m-1}$ in $F_{m-1}$ is

$$
j \frac{v_{1}-v_{2}}{2}+i \frac{v_{1}+v_{2}}{2}
$$


and the coefficient of $p^{h+\frac{n}{2}-1} q^{2 h-1}$ in $F_{n-1}^{\prime}$ is

$$
h\left(v_{1}-1\right)
$$

If we equate (58) and (59), we obtain the expected relation (2). Thus Proposition 2.1 is proved.

Corollary 2.5. The vector $\mathbf{v}$ in Proposition 2.1 does not belong to the set $\Gamma-W_{1}$

Proof. It is sufficient to prove that, for arbitrary non-negative integers $h, i, j$ such that $i+j \geq 1$, the complex line in $\mathbf{C}^{2}$

$$
i\left(v_{1}+v_{2}\right)+j\left(v_{1}-v_{2}\right)+2 h\left(1-v_{1}\right)=0
$$

does not intersect $\Gamma-W_{1}$. Assume the contrary. Then there exist nonnegative integers $h, i, j$ and a vector $\mathbf{v}=\left(v_{1}, v_{2}\right) \in \Gamma-W_{1}$ such that $i+j \geq 1$ and the relation (60) holds. Therefore we have

$$
i \Re\left(v_{1}+v_{2}\right)+j \Re\left(v_{1}-v_{2}\right)+2 h \Re\left(1-v_{1}\right)=0
$$

and

$$
i \Im\left(v_{1}+v_{2}\right)+j \Im\left(v_{1}-v_{2}\right)+2 h \Im\left(-v_{1}\right)=0 .
$$

The rest of the proof is divided into three cases:

(i) Assume that the two real parts $\Re\left(v_{1}+v_{2}\right)$ and $\Re\left(v_{1}-v_{2}\right)$ are not equal to zero. Since $\mathbf{v} \in \Gamma$, we have $\Re\left(v_{1}+v_{2}\right)>0, \Re\left(v_{1}-v_{2}\right)>0$, $\Re\left(1-v_{1}\right) \geq 0$. Hence we obtain $i=j=2 h \Re\left(1-v_{1}\right)=0$ from (61). This contradicts the condition $i+j \geq 1$.

(ii) Asuume that one of the real parts $\Re\left(v_{1}-v_{2}\right), \Re\left(v_{1}+v_{2}\right)$ is equal to zero and the other is not. Let us assume, for example, $\Re\left(v_{1}+v_{2}\right)=0$ and $\Re\left(v_{1}-v_{2}\right) \neq 0$, because we can treat the opposite case similarly. Since $\mathbf{v} \in \Gamma$, we have $\Re\left(v_{1}-v_{2}\right)>0, \Re\left(1-v_{1}\right)>0$. It follows from (61) that $j=h=0$. Since $\mathbf{v} \notin W_{1}$, we have $\Im\left(v_{1}+v_{2}\right)>0$. Therefore we have $i=0$ by (62). This contradicts $i+j \geq 1$.

(iii) Assume that $\Re\left(v_{1}-v_{2}\right)=\Re\left(v_{1}+v_{2}\right)=0$, i.e., $\Re\left(v_{1}\right)=\Re\left(v_{2}\right)=0$. Then we have $h=0$ by (61). Since $\mathbf{v} \notin W_{1}$, we have $\Im\left(v_{1}+v_{2}\right)>0$, $\Im\left(v_{1}-v_{2}\right)>0$. Therefore it follows from (62) that $i=j=0$, and this is a contradiction. 


\section{§3. Determination of invariant ideals}

We determine all the non-trivial $X(\mathbf{v})$-invariant principal ideals of $K[p, q]$ for $\mathbf{v} \in \Gamma \cap W_{1}$. This brings us the determination of classical solutions of $S(\mathbf{v})$ for $\mathbf{v} \in \Gamma \cap W_{1}$.

LEMMA 3.1. (i) Let $\mathbf{v}_{1}$ be a non-zero vector in $\Gamma \cap\left\{\mathbf{v} \in \mathbf{C}^{2} \mid v_{1}-v_{2}=\right.$ $0\}$. For every positive integer $j$, a principal ideal $\left((p-1)^{j}\right)$ is $X\left(\mathbf{v}_{1}\right)$ invariant. Conversely, if $I$ is an $X\left(\mathbf{v}_{1}\right)$-invariant principal ideal properly between the zero-ideal and $K[p, q]$, then there exists a positive integer $j$ such that $I=\left((p-1)^{j}\right)$.

(ii) Let $\mathbf{v}_{2}$ be a non-zero vector in $\Gamma \cap\left\{\mathbf{v} \in \mathbf{C}^{2} \mid v_{1}+v_{2}=0\right\}$. For every positive integer $i$, a principal ideal $\left(p^{i}\right)$ is $X\left(\mathbf{v}_{2}\right)$-invariant. Conversely, if $I$ is an $X\left(\mathbf{v}_{2}\right)$-invariant principal ideal properly between the zero-ideal and $K[p, q]$, then there exists a positive integer $i$ such that $I=\left(p^{i}\right)$.

Proof. We prove only the assertion (i). We omit the proof of (ii) because we can prove (ii) similarly. Let the notation be as in Proposition 2.1. The first half of (i) is obvious. For the second half, it is sufficient to prove that the $X\left(\mathbf{v}_{1}\right)$-invariant polynomial $F$ is equal to $(p-1)^{j}$ for some positive integer $j$. We set $\mathbf{v}_{1}=\left(v_{1}, v_{2}\right)$. Since $v_{1}=v_{2}$, we have

$$
i\left(v_{1}+v_{2}\right)+2 h\left(1-v_{1}\right)=0
$$

by (2) in $\S 2$. Then we have

$$
i \Re\left(v_{1}+v_{2}\right)+2 h \Re\left(1-v_{1}\right)=0 .
$$

Since $\Re\left(v_{1}+v_{2}\right) \geq 0$ and $\Re\left(1-v_{1}\right)>0$, we get $i=h=0$ by (1) and (2). Namely we get $j \geq 1$ and $m=0$ by (1) and (30) in $\S 2$. Hence we find $F=F_{0}=(p-1)^{j}$ by $(32)$ in $\S 2$.

LEMMA 3.2. For arbitrary non-negative integers $i$ and $j$ such that $i+$ $j \geq 1$, a principal ideal $\left(p^{i}(p-1)^{j}\right)$ is $X(\mathbf{0})$-invariant. Conversely, if $I$ is an $X(\mathbf{0})$-invariant principal ideal properly between the zero-ideal and $K[p, q]$, then there exist non-negative integers $i$ and $j$ such that $i+j \geq 1$ and $I=\left(p^{i}(p-1)^{j}\right)$.

Proof. The first half is obvious. For the second half, the notation being as in Proposition 2.1, it is sufficient to prove that the $X(\mathbf{0})$-invariant polynomial $F$ is equal to $p^{i}(p-1)^{j}$ for some non-negative integers $i$ and $j$ such that $i+j \geq 1$. Since $\mathbf{v}=\mathbf{0}$, we get $h=0$ by (2) in $\S 2$. Namely we get $m=0$ by (30) in $\S 2$. Hence we have $F=F_{0}=p^{i}(p-1)^{j}$ by (32) in $\S 2$. Here we notice that the non-negative integers $i$ and $j$ satisfy (1) in $\S 2$. 


\section{$\S 4$. Proof of Theorem 1.3}

The derivation $X(\mathbf{v})$ for $\mathbf{v} \in \Gamma-W_{1}$ satisfies the condition (J) by Corollary 2.5. By Theorem 1.1 in [23] we see that every transcendental solution $(p, q)$ of $S(\mathbf{v})$ for $\mathbf{v} \in \Gamma-W_{1}$ is non-classical.

On the other hand, by Lemmas 3.1 and 3.2 and the same argument as in Subsection 2.3 in [23], all the transcendental classical solutions of $S(\mathbf{v})$ for $\mathbf{v} \in \Gamma \cap W_{1}$ are determined by the principal prime ideals $(p)$ and $(p-1)$, and the other transcendental solutions of $S(\mathbf{v})$ for $\mathbf{v} \in \Gamma \cap W_{1}$ are not classical. Thus we complete the proof of Theorem 1.3.

\section{REFERENCES}

[1] N. Bourbaki, Groupes et algèbres de Lie, Chapitres 4, 5, et 6, Masson.

[2] B. Gambier, Sur les équations différentielles du second ordre et du premier degré dont l'intégrale générale est à points critiques fixes, Acta Math., 33 (1909), 1-55.

[3] A. S. Fokas and M. J. Ablowitz, On a unified approach to transformations and elementary solutions of Painlevé equations, J. Math. Phys., 23 (1982), 2033-2042.

[4] V. I. Gromak, Solutions of the third Painlevé equation, Diff. Eq., 9 (1973), 1599-1600.

[5] V. I. Gromak, Theory of Painlevé's equation, Diff. Eq., 11 (1975), 285-287.

[6] V. I. Gromak, One-parameter systems of solutions of Painlevé equations, Diff. Eq., 14 (1978), 1510-1513.

[7] V. I. Gromak, Reducibility of Painlevé equations, Diff. Eq., 20 (1984), 1191-1198.

[8] V. I. Gromak, Transformations of Painlevé equations, Dokl. Akad. Nauk. BSSR., 32 (1988), 395-398, (Russian).

[9] V. I. Gromak and N. A. Lukashevich, Special classes of solutions of Painlevé's equations, Diff. Eq., 18 (1982), 317-326.

[10] N. A. Lukashevich, On the theory of the third Painlevé equation, Diff. Eq., 3 (1967), 994-999.

[11] Y. Murata, Classical solutions of the third Painlevé equation, Nagoya math. J., 139 (1995), 37-65.

[12] K. Nishioka, A note on the transcendency of Painlevé's first transcendent, Nagoya Math. J., 109 (1988), 63-67.

[13] K. Okamoto, Sur les feuilletages associés aux équations du second ordre à points critiques fixes de P. Painlevé, Japan. J. Math., 5 (1979), 1-79.

[14] K. Okamoto, K, Isomonodromic deformation and Painlevé equations, and the Garnier system, J. Fac. Sci. Univ. Tokyo Sect. IA Math., 33 (1986), 575-618.

[15] K. Okamoto, Studies on the Painlevé equations IV, third Painlevé equation P PII, Funk. Ekv., 30 (1987), 305-332.

[16] P. Painlevé, Mémoire sur les équations différentielles dont l'intégrale générale est uniforme, Bull. Soc. Math. France, 28 (1900), 201-261.

[17] P. Painlevé, Sur les équations différentielles du second ordre et d'ordre supérieur dont l'intégrale est uniforme, Acta Math., 25 (1900), 1-85. 
[18] P. Painlevé, Sur les équations différentielles du second ordre à points critiques fixes, C. R. Acad. Sci. Paris, 143 (1906), 1111-1117.

[19] H. Umemura, Birational automorphism groups and differential equations, Nagoya Math. J., 119 (1990), 1-80.

[20] H. Umemura, On the irreducibility of the first differential equation of Painlevé, Algebraic Geometry and Commutative Algebra in Honor of Masayoshi NAGATA, Kinokuniya, Tokyo (1987), pp. 771-789.

[21] H. Umemura, Second proof of the irreducibility of first differential equation of Painlevé, Nagoya Math. J., 117 (1990), 125-171.

[22] H. Umemura, Differential Galois theory of infinite dimension, Nagoya Math. J., 144 (1996), 59-135.

[23] H. Umemura and H. Watanabe, Solutions of the second and fourth Painlevé equations I, Nagoya Math. J., 148 (1997), 151-198.

[24] H. Umemura and H. Watanabe, Solutions of the third Painlevé equation II, in preparation.

[25] H. Watanabe, Solutions of the fifth Painlevé equation I, Hokkaido Math. J., 24 (1995), 231-267.

Hiroshi Umemura

Graduate School of Polymathematics

Nagoya University

Chikusa-ku, Nagoya 464-8602, Japan

umemura@math.nagoya-u.ac.jp

Humihiko Watanabe

Graduate School of Mathematics

Kyushu University (at Ropponmatsu Branch)

Chuo-ku, Fukuoka 810-8560, Japan 